\title{
Maternal omega-3 fatty acid intake increases placental labyrinthine antioxidant capacity but does not protect against fetal growth restriction induced by placental ischaemia-reperfusion injury
}

\author{
Megan L Jones, Peter J Mark and Brendan J Waddell \\ School of Anatomy, Physiology and Human Biology, The University of Western Australia, 35 Stirling Highway, \\ Crawley, Western Australia 6009, Australia
}

Correspondence should be addressed to B J Waddell; Email: brendan.waddell@uwa.edu.au

\begin{abstract}
Placental oxidative stress plays a key role in the pathophysiology of several placenta-related disorders. Oxidative stress occurs when excess reactive oxygen species (ROS) damages cellular components, an outcome limited by antioxidant enzymes; mitochondrial uncoupling protein 2 (UCP2) also limits ROS production. We recently reported that maternal dietary omega-3 polyunsaturated fatty acid (n-3 PUFA) supplementation reduced placental oxidative damage and enhanced fetal and placental growth in the rats. Here, we examined the effect of n-3 PUFAs on placental antioxidant defences and whether n-3 PUFA supplementation could prevent growth restriction induced by placental ischaemia-reperfusion (IR), a known inducer of oxidative stress. Rats were fed either standard or high-n-3 PUFA diets from day 1 of pregnancy. Placentas were collected on days 17 and 22 in untreated pregnancies (term=day 23) and at day 22 following IR treatment on day 17. Expression of several antioxidant enzyme genes (Sod1, Sod2, Sod3, Cat, Txn1 and Gpx3) and Ucp2 was measured by quantitative RT-PCR in the placental labyrinth zone (LZ) and junctional zone (JZ). Cytosolic superoxide dismutase (SOD), mitochondrial SOD and catalase (CAT) activities were also analyzed. Maternal $\mathbf{n}-3$ PUFA supplementation increased LZ mRNA expression of $C$ at at both gestational days (2- and 1.5-fold respectively; $P<0.01$ ) and female $\operatorname{Sod} 2$ at day 22 (1.4-fold, $P<0.01$ ). Cytosolic SOD activity increased with n-3 PUFA supplementation at day $22(1.3$-fold, $P<0.05)$. Sod1 and Txn1 expression decreased marginally (30 and $22 \%, P<0.05)$. JZ antioxidant defences were largely unaffected by diet. Despite increased LZ antioxidant defences, maternal n-3 PUFA supplementation did not protect against placental IR-induced growth restriction of the fetus and placental LZ.

Reproduction (2013) 146 539-547
\end{abstract}

\section{Introduction}

The developing fetus requires substantial amounts of fatty acids to support rapid cellular growth and activity, and among these, the omega- 3 polyunsaturated fatty acids (n-3 PUFAs) are particularly important (Haggarty 2010). Maternal dietary supplementation with $n-3$ PUFAs has beneficial effects on pregnancy outcomes, including increased gestation length (Szajewska et al. 2006), reduced risk of pregnancy complications (Olsen et al. 2000, Oken et al. 2007, Zhou et al. 2012), and increased fetal growth (Olsen et al. 1990, Jones et al. 2013b). Potentially, n-3 PUFAs may exert beneficial effects via their involvement in several physiological pathways, including anti-oxidative pathways. Indeed, we recently reported that maternal dietary n-3 PUFA supplementation reduced placental oxidative damage and increased fetal and placental growth in rats (Jones et al. 2013b).

Oxidative stress occurs when cellular production of reactive oxygen species (ROS), by-products of cellular respiration, exceeds the protective capacity of local antioxidant defences and thus damages cellular components (Burton \& Jauniaux 2011). ROS levels are limited by a range of antioxidant enzymes including the superoxide dismutases (SODs), which catalyze conversion of superoxide to $\mathrm{H}_{2} \mathrm{O}_{2}$ and oxygen. Three isoforms of the SOD enzyme exist: cytosolic SOD1 and extracellular SOD3 are $\mathrm{Cu} / \mathrm{Zn}$ dependent, whereas mitochondrial SOD2 is $\mathrm{Mn}$ dependent. $\mathrm{H}_{2} \mathrm{O}_{2}$, itself a strong oxidizer, may then be inactivated by catalase (CAT), glutathione peroxidase (GPX) and/or the thioredoxin (TXN) system (Burton \& Jauniaux 2011). Alternatively, oxidative damage may be limited by inhibition of ROS production. For example, it has been proposed that uncoupling proteins (UCPs) may limit ROS generation by uncoupling oxidative phosphorylation (Mailloux \& Harper 2011), a primary metabolic source of ROS. Oxidative stress is commonly associated with inflammation and vice versa, as pro-inflammatory cytokines are produced in response to ROS and 
subsequently stimulate further ROS production by target cells (Burton \& Jauniaux 2011).

Placental ROS generation is high due to the high metabolic activity of placental cells (Myatt \& Cui 2004), and so all major antioxidant systems are present within the placenta (Myatt \& Cui 2004, Perkins 2006, Jones et al. 2010). Several placenta-related disorders such as pre-eclampsia $(\mathrm{PE})$, intrauterine growth restriction (IUGR), miscarriage, and gestational diabetes mellitus are characterized by increased placental oxidative stress (Coughlan et al. 2004, Biri et al. 2006, 2007, Jauniaux et al. 2006, Myatt 2010). In the case of PE, shallow implantation leads to intermittent placental blood flow through retention of vascular reactivity (Burton \& Jauniaux 2011). This predisposes the placenta to ischaemia-reperfusion (IR) injury and is associated with heightened oxidative stress and inflammation (Burton \& Jauniaux 2011). An experimental rat model of placental IR injury, termed 'uterine artery occlusion' (UAO), increases placental oxidative damage (Ishimoto et al. 1997, Nagai et al. 2008) and inhibits fetal growth (Ishimoto et al. 1997, Nakai et al. 2002, Thaete \& Neerhof 2006, Yamazaki et al. 2006, Nagai et al. 2008).

In this study, we tested the hypothesis that maternal dietary n-3 PUFA supplementation increases placental antioxidant defences in an otherwise normal pregnancy. Placental mRNA expression and activity of the major antioxidant enzymes and $U_{c p} 2$ were measured at days 17 and 22 of rat gestation (term = day 23), covering the major period of fetal growth. We also examined whether maternal n-3 PUFA supplementation could prevent placental IR-induced fetal growth restriction, potentially via anti-oxidative and anti-inflammatory mechanisms.

\section{Materials and methods}

\section{Animals and diets}

Nulliparous albino Wistar rats, 8-12 weeks old, were obtained from the Animal Resources Centre (Murdoch, WA, Australia) and maintained under controlled conditions as described previously (Hewitt et al. 2006). Rats were mated overnight, with day 1 of pregnancy designated as the day on which spermatozoa were present in a vaginal smear. On day 1 of pregnancy, mothers were placed on either a standard (Std) or high n-3 PUFA (Hn3) iso-caloric semipure diet (Specialty Feeds, Glenn Forrest, WA, Australia). Both diets consisted of $5 \%$ total fat; detailed fatty acid composition of the diets is described by Jones et al. (2013b). Mothers were either left untreated or placental IR was induced on day 17 of gestation (see below). All procedures involving animals were conducted under approval by the Animal Ethics Committee of The University of Western Australia.

\section{Placental IR induction by UAO}

On day 17 of gestation, mothers were anaesthetized by i.p. injection of $40 \mathrm{mg} / \mathrm{kg}$ body weight (BW) ketamine (Parnell
Laboratories, Sydney, NSW, Australia) and $0.05 \mathrm{mg} / \mathrm{kg}$ BW medetomidine hydrochloride (Pfizer). UAO was conducted according to the technique of Tanaka et al. (1994). Briefly, midline abdominal incisions $(\sim 3 \mathrm{~cm})$ were made sequentially in the skin and muscle layers, and the uterine horns exposed. Microvascular clamps (Fine Science Tools, North Vancouver, BC, Canada) were placed on the lower and upper ends of uterine vessels of the right uterine horn for $30 \mathrm{~min}$, during which time the vasculature visually darkened as oxygen was depleted. This effect was notably isolated to the occluded area. Body temperature was closely monitored and regulated to $37^{\circ} \mathrm{C}$ via a warming pad and infra-red warming lamp. Further heat loss was minimized by placement of temporary sutures in the skin layer to close the incision. Following removal of the microvascular clamps, the muscle and skin layers were sutured separately. Animals were then administered the analgesic, buprenorphine hydrochloride $(0.01 \mathrm{mg} / \mathrm{kg}$ BW, s.c.; Reckitt Benckiser, West Ryde, NSW, Australia) and $10 \mathrm{ml} 0.18 \%$ sterile saline with $4 \%$ glucose for rehydration. Atipamezole hydrochloride $(0.5 \mathrm{mg} / \mathrm{kg}$ BW; medetomidine hydrochloride reversal agent; Pfizer) was administered by i.m. injection to assist in recovery from anaesthesia. Control animals underwent a 'Sham' procedure, which was identical to the UAO procedure, except that microvascular clamps were not placed on uterine vessels.

\section{Tissue collection}

Rats were anaesthetized with isoflurane/nitrous oxide at either day 17 (untreated pregnancies only) or day 22 (untreated pregnancies and placental IR study) of gestation. For untreated pregnancies, three fetus-placenta pairs were obtained from the mid-region of each uterine horn (total of six per mother) and weighed. For IR-treated pregnancies (Sham and UAO), six fetus-placenta pairs were obtained from the right uterine horn in Sham-treated mothers, six fetus-placenta pairs were obtained from the 'occluded' (right) uterine horn for UAOtreated mothers, and six from the 'contralateral' (left) uterine horn. Each placenta was then dissected into labyrinth zone (LZ) and junctional zone (JZ), which were weighed individually and snap frozen in liquid nitrogen. Measurement of genes that are differentially expressed between placental zones confirms adequate zonal separation by this method (Mark et al. 2009). A blood sample obtained from the maternal dorsal aorta was mixed with 10:1 (vol:vol) 0.6 M EDTA and centrifuged at $13000 \mathrm{~g}$ for $6 \mathrm{~min}$ to obtain plasma. All tissues and plasma samples were snap frozen in liquid nitrogen and stored at $-80{ }^{\circ} \mathrm{C}$ until further analysis. Fetal sex was determined by PCR amplification of the Sry gene in day 17 fetuses as described previously (Jones et al. 2013b), and at day 22 by measuring anogenital distance (Imperato-McGinley et al. 1986).

\section{RNA and CDNA sample preparation}

Total RNA was isolated from placental zones using Tri-Reagent (Molecular Resources Centre, Cincinnati, OH, USA) as per the manufacturer's instructions. Total RNA $(1 \mu \mathrm{g})$ was used as a template for cDNA synthesis by murine Moloney leukemia virus Reverse Transcriptase RNase H Point Mutant and random 
hexamer primers (Promega) as per the manufacturer's instructions. The resultant $\mathrm{CDNAs}$ were purified using the Ultraclean PCR Cleanup kit (MoBio Industries, Solana Beach, CA, USA).

\section{Real-time quantitative RT-PCR}

Analyses of mRNA expression levels for the antioxidant genes Sod1, Sod2, Sod3, Cat, Txn1, Gpx3, and Ucp2 for the proinflammatory genes tumor necrosis factor $\alpha(T n f)$ and prostaglandin-endoperoxide synthase-2 (Ptgs2) and for the reference genes Ppia, Sdha, and Ywhaz were performed by real-time RT-PCR on the Rotorgene 6000 (Corbett Industries, Sydney, NSW, Australia) using $0.2 \mu \mathrm{M}$ of primers, SYBR Green (Molecular Probes, Eugene, OR, USA) at 1/40 000 of stock, and $0.25 \mathrm{U}$ of Immolase DNA polymerase (Bioline, Alexandria, NSW, Australia) per reaction. Primer pairs for all genes of interest (Table 1) were designed using Primer-BLAST (http:// www.ncbi.nlm.nih.gov/tools/primer-blast (Rozen \& Skaletsky 2000)). Each of the selected primer pairs were positioned to span introns to ensure no product was amplified from genomic DNA, and the resulting amplicons were sequenced to confirm specificity. Standard curves for each amplicon were generated with tenfold serial dilutions of gel-extracted (QIAEX II, Qiagen) PCR products using the Rotorgene 6000 Software. All samples were normalized against Ppia, Sdha and Ywhaz using the GeNorm algorithm (Vandesompele et al. 2002).

\section{Placental antioxidant enzyme activity}

Activities of SOD and CAT were measured in placental zone homogenates of untreated pregnancies using commercial assay kits (Cat \#706002 and 707002 respectively; Cayman Chemical
Company, Ann Arbor, MI, USA). LZ and JZ tissues ( $40 \mathrm{mg}$ of each) were homogenized using the POLYTRON-Aggregate (Kinematica, Lucerne, Switzerland). For the SOD activity assay, tissues were homogenized in 10 vol of 20 mM HEPES buffer $(\mathrm{pH}$ 7.2, containing $1 \mathrm{mM}$ EGTA, $210 \mathrm{mM}$ mannitol and $70 \mathrm{mM}$ sucrose). Samples were initially centrifuged at $1500 \mathrm{~g}$ for $5 \mathrm{~min}$ at $4{ }^{\circ} \mathrm{C}$, and the cell lysate supernatant was again centrifuged at $10000 \mathrm{~g}$ for $15 \mathrm{~min}$ to obtain aqueous and pelleted portions; the aqueous phase contained cytosolic SOD and the pelleted portion contained mitochondrial SOD. The pelleted portion was resuspended in $30 \mu \mathrm{l} 20 \mathrm{mM}$ HEPES buffer $(\mathrm{pH} 7.2$, containing $1 \mathrm{mM}$ EGTA, $210 \mathrm{mM}$ mannitol and $70 \mathrm{mM}$ sucrose). For the CAT activity assay, tissues were homogenized in $10 \mathrm{vol} 50 \mathrm{mM}$ potassium phosphate buffer $(\mathrm{pH} 7.0$, containing $1 \mathrm{mM}$ EDTA) and then centrifuged at $10000 \mathrm{~g}$ for $15 \mathrm{~min}$ at $4{ }^{\circ} \mathrm{C}$ and the cell lysate supernatant was obtained. All samples were stored at $-80^{\circ} \mathrm{C}$ until they were assayed for protein concentration by Bradford assay. Activity assays were performed as per the manufacturer's instructions and read at either 440 or $540 \mathrm{~nm}$ (SOD and CAT activity assays respectively) on the Labsystems Multiskan plate reader (Labsystems, Vantaa, Finland). Values are expressed as units per gram protein.

\section{Measurement of $F_{2}$-isoprostanes}

Levels of $\mathrm{F}_{2}$-isoprostanes were measured in female placental LZ samples of IR-treated animals. Tissues $(\sim 50 \mathrm{mg})$ were ground to a fine powder with a porcelain mortar and pestle chilled with liquid nitrogen. $\mathrm{F}_{2}$-isoprostanes were extracted and then measured in duplicate by gas chromatography-mass spectrometry using electron capture chemical ionization as described previously (Barden et al. 2012).

Table 1 Primers and PCR conditions used to measure rat placental expression of antioxidant genes, Ucp2, pro-inflammatory genes, and reference genes by real-time RT-PCR.

\begin{tabular}{|c|c|c|c|c|}
\hline Gene & Forward/reverse primer & $\begin{array}{c}\text { Annealing } \\
\text { temperature }\left({ }^{\circ} \mathrm{C}\right)\end{array}$ & $\begin{array}{l}\text { Amplicon } \\
\text { size (bp) }\end{array}$ & $\begin{array}{r}\mathrm{MgCl}_{2} \\
(\mathrm{mM})\end{array}$ \\
\hline Sod1 & $\begin{array}{l}\text { F: 5'-CGTCATTCACTTCGAGCAGA-3' } \\
\text { R: 5'-AAAATGAGGTCCTGCAGTGG-3' }\end{array}$ & 60 & 145 & 3 \\
\hline Sod2 & $\begin{array}{l}\text { F: 5'-GGCCAAGGGAGATGTTACAA-3' } \\
\text { R: 5'-GCTTGATAGCCTCCAGCAAC-3' }\end{array}$ & 60 & 149 & 3 \\
\hline Sod3 & $\begin{array}{l}\text { F: 5'-TCAGAGGCTCTTTCTCAGGC-3' } \\
\text { F: 5'-CTGCTAAGTCGACACCGGAC-3' }\end{array}$ & 60 & 195 & 2 \\
\hline Cat & $\begin{array}{l}\text { F: 5'-ACATGGTCTGGGACTTCTGG-3' } \\
\text { R: 5'-CAAGTTTTTGATGCCCCTGGT-3' }\end{array}$ & 61 & 197 & 3 \\
\hline Txn1 & $\begin{array}{l}\text { F: 5'-AGCTGATCGAGAGCAAGGAA-3' } \\
\text { R: } 5^{\prime} \text {-TCAAGGAACACCACATTGGA-3' }\end{array}$ & 60 & 160 & 3 \\
\hline Gpx3 & $\begin{array}{l}\text { F: 5'-GGCTTTGTGCCTAATTTCCA-3' } \\
\text { R: 5'-CCCACCAGGAACTTCTCAAA-3' }\end{array}$ & 60 & 188 & 3 \\
\hline Ucp2 & $\begin{array}{l}\text { F: 5'-TCATCACTTTCCCTCTAGACACC-3' } \\
\text { R: 5'-AAGCTCATCTGGCGCTGTAG-3' }\end{array}$ & 57 & 190 & 2 \\
\hline Tnf & $\begin{array}{l}\text { F: 5'-TACTGAACTTCGGGGTGATTGGTCC-3' } \\
\text { R: } 5^{\prime} \text {-CAGCCTTGTCCCTTGAAGAGAACC-3' }\end{array}$ & 60 & 295 & 3 \\
\hline Ptgs 2 & $\begin{array}{l}\text { F: } 5^{\prime} \text { GAAGGGACACCCTTTCACAT } 3^{\prime} \\
\text { R: } 5^{\prime} \text {-TGGGGAGACCATGGTAGAAC-3' }\end{array}$ & 59 & 178 & 4 \\
\hline Ppia & $\begin{array}{l}\text { F: 5'-AGCATACAGGTCCTGGCATC-3' } \\
\text { R: } 5^{\prime} \text {-TTCACCTTCCCAAAGACCAC-3' }\end{array}$ & 62 & 127 & 3 \\
\hline Sdha & $\begin{array}{l}\text { F: 5'-TGGGGCGACTCGTGGCTTTC-3' } \\
\text { R: 5'-CCCCGCCTGCACCTACAACC-3' }\end{array}$ & 60 & 134 & 2 \\
\hline Ywhaz & $\begin{array}{l}\text { F: 5'-GACGGAGCTGAGGGACATCTGC-3' } \\
\text { R: 5'-GGCTGCGAAGCATTGGGGATCA-3' }\end{array}$ & 60 & 75 & 2 \\
\hline
\end{tabular}




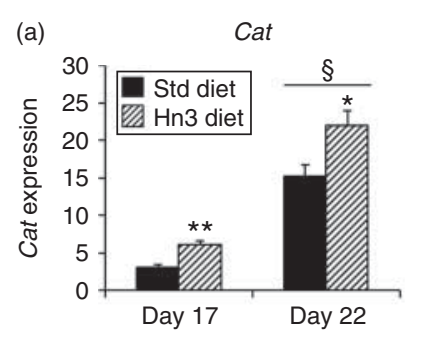

(b)
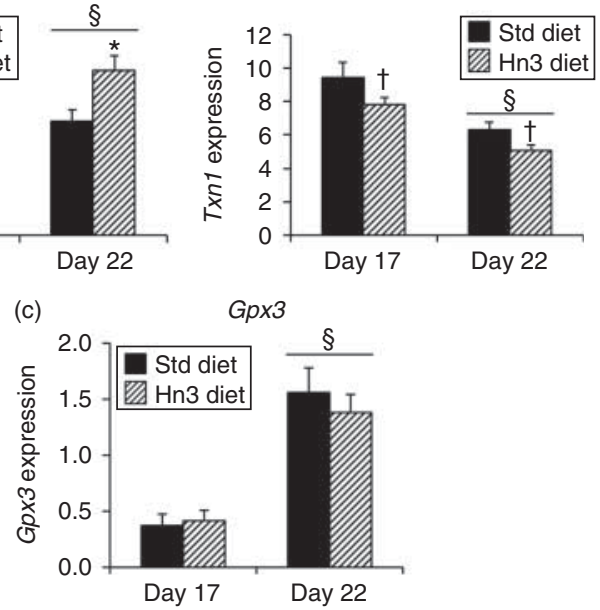

Figure 1 Labyrinth zone expression of (a) Cat, (b) Txn1, and (c) Gpx3 mRNAs at days 17 and 22 of pregnancy (fetal sex pooled). Mothers were fed either a Std or Hn3 diet from day 1 of pregnancy. Values are mean \pm S.E.M. ( $n=7-8$ per group). $* P<0.01$ and $* * P<0.001$ compared with corresponding Std diet group (two-way ANOVA and post hoc LSD tests or unpaired $t$-test); ${ }^{+} P<0.05$ overall compared with Std diet (three-way ANOVA) and ${ }^{\S} P<0.001$ compared with day 17 (three-way ANOVA).

\section{Pro-inflammatory cytokine quantitation}

Levels of the pro-inflammatory cytokines, tumor necrosis factor $\alpha$ (TNFa), interleukin 6 (IL6) and IL1b, were measured in maternal plasma of IR-treated animals using MILLIPLEX MAP Kit; Rat Cytokine/Chemokine (Cat \#RCYTO-80K; Merck Millipore, Billerica, MA, USA). Samples were centrifuged at $13000 \mathrm{~g}$ for $5 \mathrm{~min}$ prior to analysis. The assay was performed as per the manufacturer's instructions and read on CS1000 Autoplex Analyzer (PerkinElmer, Waltham, MA, USA), using Luminex xPONENT program (Luminex, TX, USA).

\section{Statistical analysis}

All analyses were conducted using Genstat version 14 (VSN International Ltd., Hemel Hempstead, UK). Where data were not normally distributed (based on residual plot analyses), values were log transformed prior to statistical analysis. In all instances, ' $n$ ' refers to the number of litters analyzed. Variation in placental SOD activity was assessed by five-way ANOVA, with variation attributed to subcellular location (cytosolic or mitochondrial), maternal diet, gestational age, placental zone, and fetal sex. Variation in CAT activity and placental gene expression was assessed by four-way ANOVA, with variation attributed to maternal diet, gestational age, placental zone, and fetal sex. Variation in plasma levels of pro-inflammatory cytokines was assessed by two-way ANOVA, with variation attributed to maternal diet and gestational age. For all ANOVAs, when a significant interaction was seen between sources of variation, separate comparisons were conducted by four-, three-, two-way ANOVA, and/or unpaired $t$-tests as appropriate. Where the $F$ test reached statistical significance $(P<0.05)$, subsequent post hoc analyses were performed using least significant difference (LSD) tests (Snedecor \& Cochran 1989).

\section{Results}

We have previously reported that maternal n-3 PUFA supplementation in this same cohort of animals increased fetal $(6 \%)$ and placental $(12 \%)$ weights at day 22 , the latter attributable primarily to enhanced growth of the LZ (Jones et al. 2013b). Furthermore, levels of the oxidative stress marker, $F_{2}$-isoprostanes, were reduced in both the placental LZ (31 and $11 \%$ at days 17 and 22 respectively) and JZ (29\% lower at day 22$)$ with the $\mathrm{Hn} 3$ diet (Jones et al. 2013b).

\section{Effects of n-3 PUFAs on placental antioxidant gene expression}

\section{Labyrinth zone}

The maternal Hn3 diet increased LZ expression of Cat at both day 17 (twofold, $P<0.001)$ and day $22(1.5$-fold, $P<0.01$; Fig. 1a) and Sod2 expression in female LZ at day 22 (1.4-fold; $P<0.01$; Fig. 2b); male Sod2 expression was unaffected by $\mathrm{Hn} 3$ diet (data not shown). By contrast, Sod1 and Txn1 expression decreased overall in response to maternal $\mathrm{Hn} 3$ diet $(30 \%, P<0.05$ and $22 \%, P<0.05$; Fig. $1 \mathrm{a}$ and $2 \mathrm{~b})$, and LZ expression of Sod3, Gpx3, and Ucp2 was unaffected.

LZ antioxidant enzyme expression varied with gestational age (Figs 1, 2 and 3). Most notably, mRNA expression of Cat, Gpx3 and Ucp2 increased from days 17 to 22 (pooled diet groups; all $P<0.001$ ). Conversely, Sod1 and Txn1 decreased over the same period

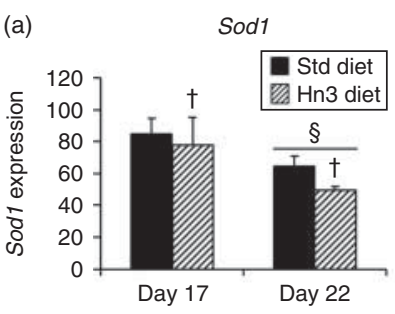

(c)

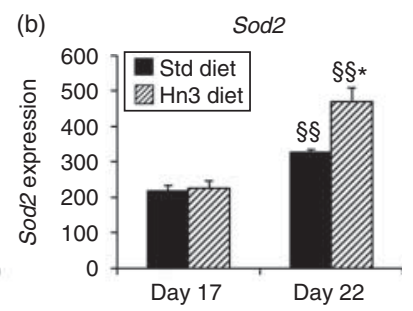

Sod3

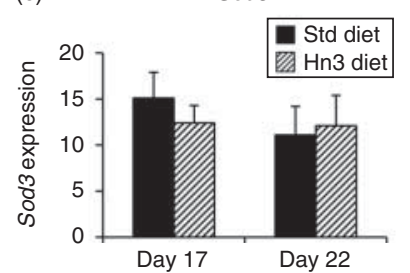

Figure 2 Labyrinth zone expression of (a) Sod1, (b) Sod2 (female only), and (c) Sod3 mRNAs at days 17 and 22 of pregnancy (fetal sex pooled except for Sod2). Mothers were fed either a Std or $\mathrm{Hn} 3$ diet from day 1 of pregnancy. Male Sod 2 data is not presented because expression was unaffected by maternal diet. Values are mean \pm S.E.M. $(n=7-8$ per group). ${ }^{*} P<0.01$ compared with corresponding Std diet group (two-way ANOVA and post hoc LSD tests or unpaired $t$-test); ${ }^{+} P<0.05$ overall compared with Std diet (three-way ANOVA); ${ }^{\S} P<0.01$ overall compared with day 17 (pooled diet groups, three-way ANOVA) and ${ }^{\S} P<0.001$ compared with day 17 value of same diet group (unpaired $t$-test). 


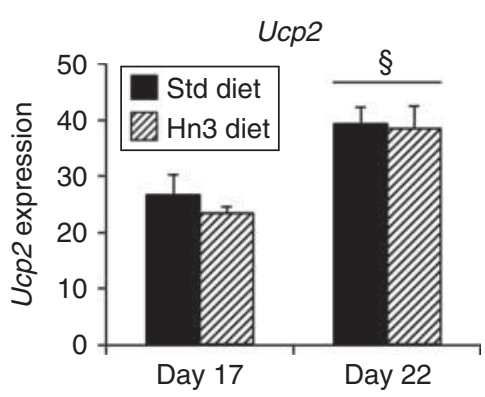

Figure 3 Labyrinth zone expression of Ucp2 mRNAs at days 17 and 22 of pregnancy (fetal sex pooled). Mothers were fed either a Std or Hn3 diet from day 1 of pregnancy. Values are mean \pm s.E.M. $(n=7-8$ per group). ${ }^{\S} P<0.001$ compared with day 17 (three-way ANOVA).

(overall, $P<0.01$ and $P<0.001$ ), whereas that of Sod3 did not vary with gestational age. These gestational age effects were unaffected by $\mathrm{Hn} 3$ diet, with the exception of Sod2 in females, where a significant interaction term between gestational age and diet was observed $(P<0.01)$. Specifically, the Sod2 gestational increase from days 17 to 22 was greater in the $\mathrm{Hn} 3$ group (2.1-fold increase, $P<0.001)$ than the Std group (1.5-fold increase, $P<0.001$; Fig. $2 \mathrm{~b}$ ).

\section{Junctional zone}

JZ mRNA expression of antioxidant enzymes and Ucp2 was largely unaffected by the $\mathrm{Hn} 3$ diet, with the exception of Txn1, which increased in female $\mathrm{JZ}$ at day 17 (2.5-fold, $P<0.01)$, and Sod3, which decreased overall (both gestational days, $42-45 \%, P<0.001$ ) (Supplementary Figures 1, 2 and 3, see section on supplementary data given at the end of this article). JZ expression of antioxidant genes was generally higher in male compared with female samples, most notably for Sod2, Cat, and Txn1 (see Supplementary Figures 1 and 2). Despite this, diet and gestational age effects were similar in the JZ of males and females.

JZ expression of several antioxidant enzymes changed with gestational age and these shifts were variably affected by the maternal Hn3 diet (Supplementary Figures 1, 2 and 3). For example, while the $\mathrm{Hn} 3$ diet dampened gestational increases in both Sod1 and Txn1 expression in males, it increased Cat expression over this period in both sexes (each $P<0.001$ ). Moreover, the gestational decline in JZ Txn1 expression in females was enhanced by the $\mathrm{Hn} 3$ diet $(P<0.05)$.

\section{Effects of n-3 PUFAs on placental SOD and CAT enzyme activities}

Placental levels of SOD and CAT activity were similar in males and females, and so data were pooled for all further analyses. In the $\mathrm{LZ}$, the $\mathrm{Hn} 3$ diet increased cytosolic SOD activity at day $22(29 \%$; $P<0.05$; Fig. 4a) but had no effect on mitochondrial SOD and CAT activities on either day (Fig. 4b and c). In the JZ, the Hn3 diet increased cytosolic SOD activity only at day 17 $(32 \% ; P<0.01)$ and had no effect on JZ mitochondrial SOD and CAT activities (Supplementary Figure 4, see section on supplementary data given at the end of this article).

The maternal $\mathrm{Hn} 3$ diet also variably affected gestational changes (i.e. between days 17 and 22) in placental SOD and CAT activities (Fig. 4, Supplementary Figure 4). Most notably, the $\mathrm{Hn} 3$ diet prevented the gestational decline in LZ activity of cytosolic SOD (Fig. 4a), whereas JZ activity of cytosolic SOD decreased only in $\mathrm{Hn} 3$ group over the same period (15\% decrease; $P<0.05$; Supplementary Figure 4a).

\section{Placental IR}

\section{Fetal and placental weights}

UAO treatment affected growth outcomes of males and females similarly, and so data were pooled for further analysis (Fig. 5). Maternal $\mathrm{Hn} 3$ diet increased fetal weight overall (4.5\% increase, pooled treatment groups; $P<0.05)$, consistent with observed growth outcomes in untreated pregnancies (Jones et al. 2013b). In UAOtreated mothers, fetal weight of the occluded horn was reduced overall compared with Sham (pooled diet groups, $P<0.01)$, although by LSD test this was significant only in the $\mathrm{Hn} 3$ group $(7.7 \%$ reduction, $P<0.05)$, and was a strong trend in the Std diet group (8.3\% reduction, $P=0.053)$. Fetal weight of the contralateral horn was unaffected by UAO irrespective of maternal diet. In Sham-treated animals, placental weight increased with maternal $\mathrm{Hn} 3$ diet $(9 \%, P<0.05)$, again
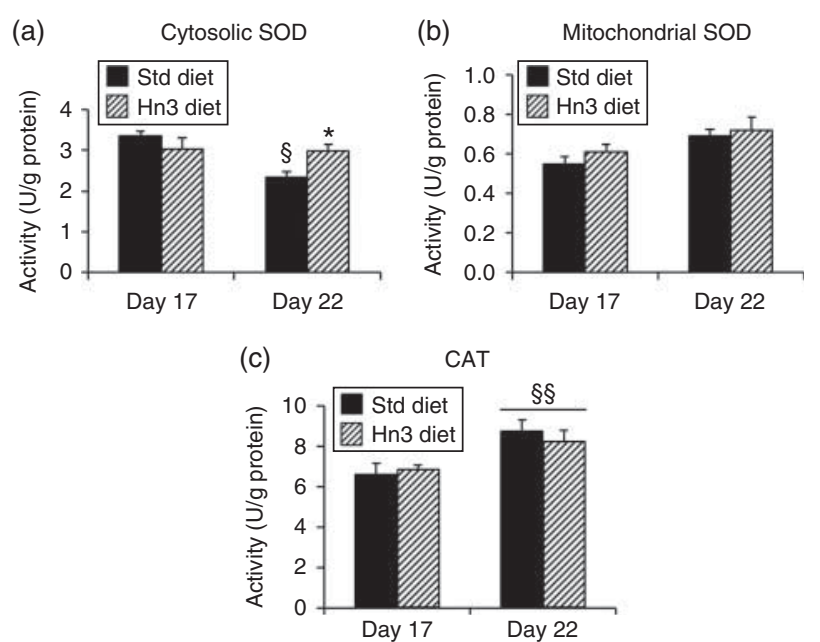

Figure 4 Labyrinth zone activity of (a) cytosolic SOD, (b) mitochondrial SOD, and (c) CAT at days 17 and 22 of pregnancy (fetal sex pooled). Mothers were fed either a Std or $\mathrm{Hn} 3$ diet from day 1 of pregnancy. Values are mean \pm S.E.M. $\left(n=6-8\right.$ per group). ${ }^{*} P<0.05$ compared with corresponding Std diet group (two-way ANOVA); ${ }^{\S} P<0.01$ and ${ }^{\S \S} P<0.001$ compared with day 17 (three- or two-way ANOVA). 

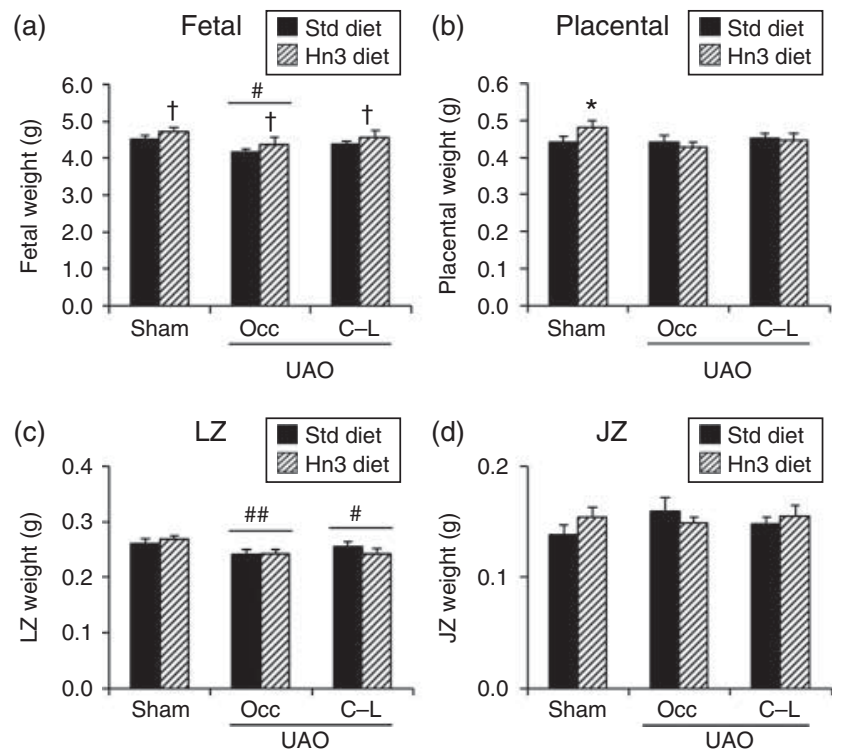

Figure 5 Fetal and placental weights in placental IR. (a) Fetal, (b) placental, (c) LZ, and (d) JZ weights at day 22 following Sham or UAO treatment (fetal sex pooled). In UAO-treated animals, weights of the occluded horn $(\mathrm{Occ})$ and contralateral horn $(\mathrm{C}-\mathrm{L})$ are considered separately. Mothers were fed either a Std or a $\mathrm{Hn} 3$ diet from day 1 of pregnancy. Values are mean \pm S.E.M. ( $n=7-8$ per group). ${ }^{\#} P<0.05$ and ${ }^{\#} P<0.01$ compared with Sham (three-way ANOVA, post hoc LSD tests); ${ }^{*} P<0.05$ compared with corresponding Std diet group (two-way ANOVA, post hoc LSD tests) and ${ }^{\dagger} P<0.05$ overall compared with Std diet (three-way ANOVA).

consistent with untreated pregnancy (Jones et al. 2010). In UAO-treated mothers, total placental weight was unaffected in both diet groups, although LZ weight was reduced in both occluded $(7.9-11.3 \%, P<0.01)$ and contralateral horns (2.5-10.9\%; overall $P<0.05$, pooled diet groups); IZ weight was unaffected by UAO. Ratios between fetal, whole placental, and LZ weights did not differ with maternal diet or UAO treatment (data not shown).

\section{Placental LZ F $F_{2}$-isoprostanes}

Levels of $F_{2}$-isoprostanes were measured in female $L Z$ samples of IR-treated mothers (Sham and UAO). Levels decreased in response to maternal $\mathrm{Hn} 3$ dietary intake $(19.4-33.6 \%$ decrease; pooled treatment groups, $P<0.05$; data not shown), consistent with untreated pregnancies (Jones et al. 2013b). UAO treatment did not affect LZ levels of $\mathrm{F}_{2}$-isoprostanes (data not shown).

\section{Maternal circulating levels and placental gene expression of pro-inflammatory mediators}

Maternal TNFa levels were not affected by either maternal $\mathrm{Hn} 3$ or UAO treatment (data not shown). Maternal circulating levels of IL1b were increased with Hn3 diet $(53 \% ; P<0.05)$, consistent with our previous observations (Jones et al. 2013a). IL6 was undetectable in maternal plasma, and placental LZ Tnfexpression was not affected by either the maternal $\mathrm{Hn} 3$ diet or the UAO treatment (Fig. 6a). Ptgs2 expression was lower overall in female compared with male LZ (overall sex effect, $P<0.01$ ), but diet and UAO treatment effects were similar for each sex. The maternal $\mathrm{Hn} 3$ diet reduced Ptgs2 expression in the Occ group of UAO-treated mothers $(P<0.05$ for each sex; Fig. 6b). Surprisingly, Ptgs2 expression was higher in Sham- than in UAOtreated LZ (occluded or contralateral; 36-49\% higher; overall treatment effect $P<0.001$; Fig. 6b). JZ expression of both Tnf and Ptgs2 was unaffected by maternal diet or UAO treatment (data not shown).

\section{Discussion}

Mounting evidence suggests that oxidative stress in uteroplacental tissues plays a key role in the development of pregnancy complications, including PE and IUGR. Maternal dietary n-3 PUFA supplementation may be a possible therapeutic intervention for these disorders, given their ability to reduce placental oxidative damage (Jones et al. 2013b). The aim of this study was to investigate the impact of maternal n-3 PUFA supplementation on the antioxidant capacity of the late gestation placenta. The major findings were that maternal n-3 PUFA supplementation enhanced the expression, and to a lesser extent activity, of various antioxidant enzymes in the placental LZ. These observations extend our earlier report that maternal dietary n-3 PUFA intake reduced placental $F_{2}$-isoprostanes (a highly reliable marker of oxidative damage) and was associated with increased fetal and placental growth (Jones et al. 2013b). Despite enhanced basal LZ levels of antioxidant enzymes and reduced placental oxidative stress in otherwise normal pregnancies, dietary n-3 PUFA supplementation could not prevent IR-induced fetal and placental growth restriction.
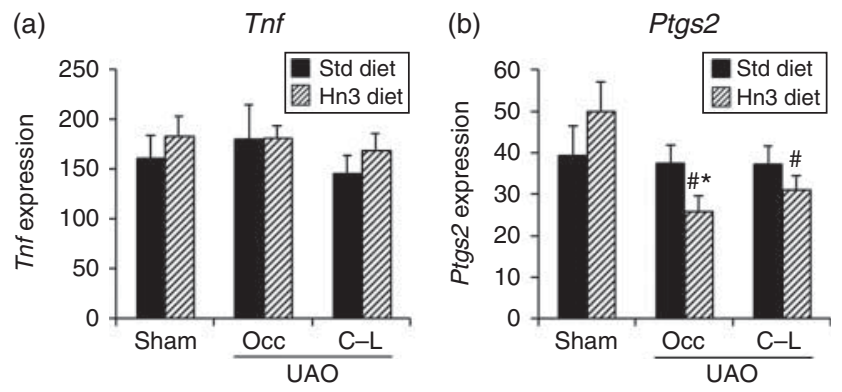

Figure 6 Labyrinth zone expression of (a) Tnf (pooled sexes) and (b) Ptgs2 (female only) at day 22 following Sham or UAO treatment. In UAO-treated animals, the occluded horn (Occ) and contralateral horn $(\mathrm{C}-\mathrm{L})$ were considered separately. Mothers were fed either a Std or a $\mathrm{Hn} 3$ diet from day 1 of pregnancy. Values are mean \pm s.E.M. $(n=7-8$ per group). ${ }^{*} P<0.05$ compared with corresponding Sham value (two-way ANOVA, post hoc LSD tests) and $* P<0.05$ compared with Std diet of same treatment group (two-way ANOVA, post hoc LSD tests). 
The importance of limiting placental oxidative stress during normal gestation has recently been shown by two studies. First, Umekawa et al. (2008) demonstrated that global TXN1 overexpression reduced oxidative damage and enhanced fetal growth in a mouse model. Similarly, we recently reported reduced placental oxidative damage and increased fetal and placental growth with maternal dietary n-3 PUFA supplementation (Jones et al. 2013b). Together, these studies suggest that placental ROS tonically suppress fetal growth during normal pregnancy. Because the placental LZ is the site of maternal-fetal exchange, enhanced antioxidant capacity of this placental zone could significantly impact on fetal growth outcomes. In this study, LZ mRNA expression of Cat was increased at both gestational days in response to maternal n-3 PUFA supplementation, as was Sod2 in female LZ at day 22. Concomitantly, expression of Sod1 and Txn1 was reduced, although these effects were marginal. These results point to a likely redundancy in the antioxidant defences, given that several antioxidant enzymes catalyze conversion of the same ROS molecule. Therefore, despite the marginal falls in Txn1 and Sod1, corresponding increases in Cat and Sod 2 mRNA expression likely account for the previously reported reduction in $\mathrm{F}_{2}$-isoprostanes (Jones et al. 2013b). Enhanced Cat expression in response to maternal Hn3 diet was not matched by higher CAT activity levels, but it is possible that this reflected lower sensitivity of the activity assay relative to quantitative PCR. Despite an overall slight reduction of Sod1 mRNA expression in the placental LZ with maternal n-3 PUFA supplementation, corresponding cytosolic SOD activity was increased at day 22, presumably reflecting increased enzyme efficiency or activation, potentially via posttranslational regulatory mechanisms. Overall, these results suggest that antioxidant capacity of the placental LZ was enhanced by maternal n-3 PUFA supplementation and may in part contribute to increased fetal and placental LZ growth (Jones et al. 2013b).

In contrast to the LZ, antioxidant responses of the JZ to maternal n-3 PUFA supplementation were minimal. While the $L Z$ grows rapidly during late gestation to meet fetal demand, the JZ regresses during this time (Waddell et al. 2000). As such, it is perhaps unsurprising that antioxidant responses differ between the two placental zones in late gestation, and this highlights the need to consider these zones separately in rodent studies. The majority of antioxidant enzymes were more highly expressed in the placental LZ than in the JZ, the single exception being Gpx3 expression, which was considerably higher in $\mathrm{JZ}$, and this is consistent with our previous findings in chow-fed animals (Jones et al. 2010). Overall, the placental LZ appeared to exhibit enhanced antioxidant defences compared with the JZ. Accordingly, levels of $F_{2}$-isoprostanes were lower in the placental LZ than in the JZ (Jones et al. 2013b).
Placental ROS accumulation may also be controlled by limiting ROS generation. Previous studies have established an important role for Ucp2 in limiting oxidative damage in other tissues, with $U_{c p}$ 2-null mice showing increased ROS production in several cell types (Arsenijevic et al. 2000, Chevillotte et al. 2007). Dietary n-3 PUFAs have been shown to increase Ucp2 mRNA expression in mouse white adipose tissue in vivo (Hun et al. 1999) and in rat myocytes in vitro (Hatakeyama \& Scarpace 2001). Despite these previous observations, maternal dietary supplementation with n-3 PUFAs did not affect placental $U_{c p} 2$ expression in this study. This may suggest that basal placental $U_{c p 2}$ expression is already relatively high in normal pregnancy and is thus unresponsive to further stimulation.

In addition to their anti-oxidative properties, the anti-inflammatory and pro-resolving actions of $n-3$ PUFAs are well documented (Calder 2012). Inflammation is closely associated with oxidative stress, given that pro-inflammatory cytokines produced in response to ROS accumulation subsequently stimulate further ROS production by target cells (Burton \& Jauniaux 2011). Furthermore, we recently reported increased placental synthesis of pro-inflammatory cytokines towards term in the rat (Mark et al. 2013). Consequently, the beneficial effects of n-3 PUFA supplementation on oxidative status could be accounted for, in part, by their anti-inflammatory actions. Although levels of proinflammatory mediators (placental and maternal) were not suppressed by n-3 PUFA supplementation, placental levels of the n-3 PUFA-derived pro-resolving mediators, resolvins and protectins, were markedly increased (Jones et al. 2013a). This could be relevant to antioxidant capacity, as administration of resolvin D1 can limit oxidative stress-induced inflammation in the mouse (Spite et al. 2009), and protectin D1 confers protection from oxidative stress-induced apoptosis in human retinal pigment epithelial cells (Faghiri \& Bazan 2010). Thus, the enhanced levels of placental resolvins and protectins induced by $n-3$ PUFA supplementation may protect against oxidative damage.

In this study, we show that expression and activity of antioxidant enzymes increase towards term, confirming our previous report in chow-fed animals (Jones et al. 2010). This is consistent with an up-regulation of placental antioxidant defences as placental oxygenation increases, which occurs on two occasions during normal gestation: following the rapid onset of maternal intraplacental circulation (10-12 weeks human gestation (Myatt \& Cui 2004)) and during late gestation when fetal demand is maximal (Takehara et al. 1990, Perkins 2006).

Omega-3 PUFA administration has also been shown to confer protection against hepatic (Kim et al. 2013), renal (Ashtiyani et al. 2012), intestinal (Arisue et al. 2012, Brahmbhatt et al. 2013), cerebral (Pan et al. 2009), pulmonary (Lee et al. 2008), and myocardial (ZeghichiHamri et al. 2010, Gao et al. 2011) IR injury. 
Furthermore, given our previous observation of reduced placental oxidative damage with maternal n-3 PUFA supplementation in normal pregnancy (Jones et al. $2013 \mathrm{~b}$ ) and increased LZ antioxidant defences as demonstrated here, we hypothesized that maternal n-3 PUFA supplementation would prevent placental IR-induced growth restriction. On the contrary, the adverse impact of IR on fetal and placental growth was similar in Std and Hn3 diet-fed mothers. This may suggest that while maternal $n-3$ PUFA supplementation is beneficial in a normal pregnancy setting, its capacity to protect against a major IR insult is limited.

UAO treatment did not affect markers of placental LZ oxidative damage $\left(\mathrm{F}_{2}\right.$-isoprostanes), maternal or placental inflammation. This may be because these were analyzed 5 days following $\cup A O$, thereby allowing time for tissue recovery and resolution of inflammation. As such it would be of interest to measure oxidative and inflammatory markers nearer to the time of UAO treatment. As fetal growth is maximal between days 17 and 22, collecting tissues at day 22 allowed us to best examine the effect of UAO on fetal and placental growth outcomes. Fetal growth was restricted by around $8 \%$ after UAO treatment, somewhat greater than that observed in other studies utilizing this model (e.g. as little as $4 \%$ fetal weight restriction 5 days after treatment (Yamazaki et al. 2006)).

In conclusion, this study demonstrates that maternal dietary supplementation with n-3 PUFAs increases the antioxidant capacity of the placental LZ, which may contribute to reduced placental oxidative status and increased fetal and placental growth outcomes (Jones et al. 2013b). Despite this increased antioxidant capacity, maternal n-3 PUFA supplementation did not protect against placental IR-induced fetal and placental LZ growth restriction.

\section{Supplementary data}

This is linked to the online version of the paper at http://dx. doi.org/10.1530/REP-13-0282.

\section{Declaration of interest}

The authors declare that there is no conflict of interest that could be perceived as prejudicing the impartiality of the research reported.

\section{Funding}

This work was supported by the National Health and Medical Research Council of Australia (grant number 572621).

\section{Acknowledgements}

The authors wish to acknowledge Prof. Trevor Mori (School of Medicine and Pharmacology, The University of
Western Australia) for analysis of $F_{2}$-isoprostanes, and the technical assistance of Ms Tracey Lee-Pullen (Centre for Microscopy, Characterization and Analysis, The University of Western Australia, Australia) for the Milliplex cytokine assay.

\section{References}

Arisue A, Shimojima N, Tomiya M, Shimizu T, Harada D, Nakayama M, Tomita H, Shinoda M, Tanabe M, Maruyama I et al. 2012 Effect of an omega-3 lipid emulsion in reducing oxidative stress in a rat model of intestinal ischemia-reperfusion injury. Pediatric Surgery International 28 913-918. (doi:10.1007/s00383-012-3144-0)

Arsenijevic D, Onuma H, Pecqueur C, Raimbault S, Manning BS, Miroux B, Couplan E, Alves-Guerra MC, Goubern M, Surwit R et al. 2000 Disruption of the uncoupling protein-2 gene in mice reveals a role in immunity and reactive oxygen species production. Nature Genetics $\mathbf{2 6}$ 435-439. (doi:10.1038/82565)

Ashtiyani SC, Najafi H, Kabirinia K, Vahedi E \& Jamebozorky L 2012 Oral omega-3 fatty acid for reduction of kidney dysfunction induced by reperfusion injury in rats. Iranian Journal of Kidney Diseases 6 275-283.

Barden AE, Corcoran TB, Mas E, Durand T, Galano J-M, Roberts LJ, Paech M, Muchatuta NA, Philips M \& Mori TA 2012 Is there a role for isofurans and neuroprostanes in pre-eclampsia and normal pregnancy? Antioxidants \& Redox Signaling 16 165-169. (doi:10.1089/ars.2011.4214)

Biri A, Onan A, Devrim E, Babacan F, Kavutcu M \& Durak Ä 2006 Oxidant status in maternal and cord plasma and placental tissue in gestational diabetes. Placenta 27 327-332. (doi:10.1016/j.placenta.2005.01.002)

Biri A, Bozkurt N, Turp A, Kavutcu M, Himmetoglu O \& Durak I 2007 Role of oxidative stress in intrauterine growth restriction. Gynecologic and Obstetric Investigation 64 187-192. (doi:10.1159/000106488)

Brahmbhatt V, Oliveira M, Briand M, Perrisseau G, Bastic Schmid V, Destaillats F, Pace-Asciak C, Benyacoub J \& Bosco N 2013 Protective effects of dietary EPA and DHA on ischemia-reperfusion-induced intestinal stress. Journal of Nutritional Biochemistry 24 104-111. (doi:10.1016/j.jnutbio.2012.02.014)

Burton GJ \& Jauniaux E 2011 Oxidative stress. Best Practice \& Research. Clinical Obstetrics \& Gynaecology 25 287-299. (doi:10.1016/ j.bpobgyn.2010.10.016)

Calder PC 2012 Long-chain fatty acids and inflammation. Proceedings of the Nutrition Society 71 284-289. (doi:10.1017/S0029665112000067)

Chevillotte E, Giralt M, Miroux B, Ricquier D \& Villarroya F 2007 Uncoupling protein-2 controls adiponectin gene expression in adipose tissue through the modulation of reactive oxygen species production. Diabetes 56 1042-1050. (doi:10.2337/db06-1300)

Coughlan MT, Vervaart PP, Permezel M, Georgiou HM \& Rice GE 2004 Altered placental oxidative stress status in gestational diabetes mellitus. Placenta 25 78-84. (doi:10.1016/S0143-4004(03)00183-8)

Faghiri Z \& Bazan NG $2010 \mathrm{PI3K/Akt} \mathrm{and} \mathrm{mTOR/p70S6K} \mathrm{pathways} \mathrm{mediate}$ neuroprotectin D1-induced retinal pigment epithelial cell survival during oxidative stress-induced apoptosis. Experimental Eye Research 90 718-725. (doi:10.1016/j.exer.2010.03.002)

Gao J, Yasuda S, Tsuburaya R, Ito Y, Shiroto T, Hao K, Aizawa K, Kikuchi Y, Ito K \& Shimokawa H 2011 Long-term treatment with eicosapentaenoic acid ameliorates myocardial ischemia-reperfusion injury in pigs in vivoinvolvement of Rho-kinase pathway inhibition. Circulation Journal 75 1843-1851. (doi:10.1253/circj.CJ-11-0209)

Haggarty P 2010 Fatty acid supply to the human fetus. Annual Review of Nutrition 30 237-255. (doi:10.1146/annurev.nutr.012809.104742)

Hatakeyama Y \& Scarpace PJ 2001 Transcriptional regulation of uncoupling protein-2 gene expression in L6 myotubes. International Journal of Obesity 25 1619-1624. (doi:10.1038/sj.ijo.0801812)

Hewitt DP, Mark PJ \& Waddell BJ 2006 Placental expression of peroxisome proliferator-activated receptors in rat pregnancy and the effect of increased glucocorticoid exposure. Biology of Reproduction 74 23-28. (doi:10.1095/biolreprod.105.045914)

Hun CS, Hasegawa K, Kawabata T, Kato M, Shimokawa T \& Kagawa Y 1999 Increased uncoupling protein2 mRNA in white adipose tissue, and decrease in leptin, visceral fat, blood glucose, and cholesterol in KK-Ay mice fed with eicosapentaenoic and docosahexaenoic acids in addition to linolenic acid. Biochemical and Biophysical Research Communications 259 85-90. (doi:10.1006/bbrc.1999.0733) 
Imperato-McGinley J, Binienda Z, Gedney J \& Vaughan EDJ 1986 Nipple differentiation in fetal male rats treated with an inhibitor of the enzyme $5 \alpha$-reductase: definition of a selective role for dihydrotestosterone. Endocrinology 118 132-137. (doi:10.1210/endo-118-1-132)

Ishimoto H, Natori M, Tanaka M, Miyazaki T, Kobayashi T \& Yoshimura Y 1997 Role of oxygen-derived free radicals in free growth retardation induced by ischemia-reperfusion in rats. American Journal of Physiology $272 \mathrm{H} 701-\mathrm{H} 705$.

Jauniaux E, Poston L \& Burton GJ 2006 Placental-related diseases of pregnancy: involvement of oxidative stress and implications in human evolution. Human Reproduction Update 12 747-755. (doi:10.1093/ humupd/dml016)

Jones ML, Mark PJ, Lewis JL, Mori TA, Keelan JA \& Waddell BJ 2010 Antioxidant defenses in the rat placenta in late gestation: increased labyrinthine expression of superoxide dismutases, glutathione peroxidase 3, and uncoupling protein 2. Biology of Reproduction 83 254-260. (doi:10.1095/biolreprod.110.083907)

Jones ML, Mark PJ, Keelan JA, Barden A, Mas E, Mori TA \& Waddell BJ 2013 a Maternal dietary omega-3 fatty acid intake increases resolvin and protectin levels in the rat placenta. Journal of Lipid Research $\mathbf{5 4}$ 2247-2254. (doi:10.1194/jIr.M039842)

Jones ML, Mark PJ, Mori TA, Keelan JA \& Waddell BJ 2013b Maternal dietary omega- 3 fatty acid supplementation reduces placental oxidative stress and increases fetal and placental growth in the rat. Biology of Reproduction 88 37. (doi:10.1095/biolreprod.112.103754)

Kim K, Jung N, Lee K, Choi J, Kim S, Jun J, Kim E \& Kim D 2013 Dietary omega-3 polyunsaturated fatty acids attenuate hepatic ischemia/reperfusion injury in rats by modulating toll-like receptor recruitment into lipid rafts. Clinical Nutrition 32 855-862. (doi:10.1016/j.clnu.2012.11.026)

Lee JC, Bhora F, Sun J, Cheng G, Arguiri E, Solomides CC, Chatterjee S \& Christofidou-Solomidou M 2008 Dietary flaxseed enhances antioxidant defenses and is protective in a mouse model of lung ischemiareperfusion injury. American Journal of Physiology. Lung Cellular and Molecular Physiology 294 L255-L265. (doi:10.1152/ajplung. 00138.2007)

Mailloux RJ \& Harper M-E 2011 Uncoupling proteins and the control of mitochondrial reactive oxygen species production. Free Radical Biology \& Medicine 51 1106-1115. (doi:10.1016/j.freeradbiomed.2011.06.022)

Mark PJ, Augustus S, Lewis JL, Hewitt DP \& Waddell BJ 2009 Changes in the placental glucocorticoid barrier during rat pregnancy: impact on placental corticosterone levels and regulation by progesterone. Biology of Reproduction 80 1209-1215. (doi:10.1095/biolreprod.108.073650)

Mark PJ, Lewis JL, Jones ML, Keelan JA \& Waddell BJ 2013 The inflammatory state of the rat placenta increases in late gestation and is further enhanced by glucocorticoids in the labyrinth zone. Placenta $\mathbf{3 4}$ 559-566. (doi:10.1016/j.placenta.2013.04.006)

Myatt L 2010 Review: Reactive oxygen and nitrogen species and functional adaptation of the placenta. Placenta 31 S66-S69. (doi:10.1016/ j.placenta.2009.12.021)

Myatt L \& Cui X 2004 Oxidative stress in the placenta. Histochemistry and Cell Biology 122 369-382. (doi:10.1007/s00418-004-0677-x)

Nagai R, Watanabe K, Wakatsuki A, Hamada F, Shinohara K, Hayashi Y, Imamura R \& Fukaya T 2008 Melatonin preserves fetal growth in rats by protecting against ischemia/reperfusion-induced oxidative/nitrosative mitochondrial damage in the placenta. Journal of Pineal Research $\mathbf{4 5}$ 271-276. (doi:10.1111/j.1600-079X.2008.00586.x)

Nakai A, Taniuchi Y, Oya A, Asakura H, Koshino T \& Araki T 2002 Windows of therapeutic opportunity on fetal growth retardation induced by transient intrauterine ischemia in rats. Journal of Nippon Medical School 69 534-541. (doi:10.1272/jnms.69.534)

Oken E, Ning Y, Rifas-Shiman SL, Rich-Edwards JW, Olsen SF \& Gillman MW 2007 Diet during pregnancy and risk of preeclampsia or gestational hypertension. Annals of Epidemiology 17 663-668. (doi:10.1016/j.annepidem.2007.03.003)

Olsen SF, Olsen JR \& Frische G 1990 Does fish consumption during pregnancy increase fetal growth? A study of the size of the newborn, placental weight and gestational age in relation to fish consumption during pregnancy International Journal of Epidemiology 19 971-977. (doi:10.1093/ije/19.4.971)

Olsen SF, Secher NJ, Tabor A, Weber T, Walker JJ \& Gluud C 2000 Randomised clinical trials of fish oil supplementation in high risk pregnancies. Fish oil trials in pregnancy (FOTIP) team. BJOG: an International Journal of Obstetrics and Gynaecology 107 382-395. (doi:10.1111/j.1471-0528.2000.tb13235.x)

Pan H-C, Kao T-K, Ou Y-C, Yang D-Y, Yen Y-J, Wang C-C, Chuang Y-H, Liao S-L, Raung S-L, Wu C-W et al. 2009 Protective effect of docosahexaenoic acid against brain injury in ischemic rats. Journal of Nutritional Biochemistry 20 715-725. (doi:10.1016/j.jnutbio. 2008.06.014)

Perkins AV 2006 Endogenous anti-oxidants in pregnancy and preeclampsia. Australian \& New Zealand Journal of Obstetrics \& Gynaecology 46 77-83. (doi:10.1111/j.1479-828X.2006.00532.x)

Rozen S \& Skaletsky H 2000 Primer3 on the WWW for general users and for biologist programmers. Methods in Molecular Biology 132 365-386.

Snedecor G \& Cochran W 1989 Statistical Methods. Ames, IA: lowa State University Press.

Spite M, Summers L, Porter TF, Srivastava S, Bhatnagar A \& Serhan CN 2009 Resolvin D1 controls inflammation initiated by glutathione-lipid conjugates formed during oxidative stress. British Journal of Pharmacology 158 1062-1073. (doi:10.1111/j.1476-5381.2009.00234.x)

Szajewska H, Horvath A \& Koletzko B 2006 Effect of n-3 long-chain polyunsaturated fatty acid supplementation of women with low-risk pregnancies on pregnancy outcomes and growth measures at birth: a meta-analysis of randomized controlled trials. American Journal of Clinical Nutrition 83 1337-1344.

Takehara Y, Yoshioka T \& Sasaki J 1990 Changes in levels of lipoperoxide and antioxidant factors in human placenta during gestation. Acta Medica Okayama 44 103-111.

Tanaka M, Natori M, Ishimoto H, Miyazaki T, Kobayashi T \& Nozawa S 1994 Fetus-placenta-newborn: experimental growth retardation produced by transient period of uteroplacental ischemia in pregnant Sprague-Dawley rats. American Journal of Obstetrics and Gynecology 171 1231-1234. (doi:10.1016/0002-9378(94)90138-4)

Thaete LG \& Neerhof MG 2006 Endothelin and platelet-activating factor: significance in the pathophysiology of ischemia/reperfusion-induced fetal growth restriction in the rat. American Journal of Obstetrics and Gynecology 194 1377-1383. (doi:10.1016/j.ajog.2005.11.019)

Umekawa T, Sugiyama T, Kihira T, Murabayashi N, Zhang L, Nagao K, Kamimoto Y, Ma N, Yodoi J \& Sagawa N 2008 Overexpression of thioredoxin-1 reduces oxidative stress in the placenta of transgenic mice and promotes fetal growth via glucose metabolism. Endocrinology 149 3980-3988. (doi:10.1210/en.2007-1682)

Vandesompele J, Preter KD, Pattyn F, Poppe B, Roy NV, Paepe AD \& Speleman F 2002 Accurate normalization of real-time quantitative RT-PCR data by geometric averaging of multiple internal control genes. Genome Biology 3 RESEARCH0034. (doi:10.1186/gb-2002-37-research0034)

Waddell BJ, Hisheh S, Dharmarajan AM \& Burton PJ 2000 Apoptosis in rat placenta Is zone-dependent and stimulated by glucocorticoids. Biology of Reproduction 63 1913-1917. (doi:10.1095/biolreprod63.6.1913)

Yamazaki K, Endo T, Kitajima Y, Manase K, Nagasawa K, Honnma H, Hayashi T, Kudo R \& Saito T 2006 Elevation of both cyclooxygenase-2 and prostaglandin E2 receptor EP3 expressions in rat placenta after uterine artery ischemia-reperfusion. Placenta 27 395-401. (doi:10.1016/ j.placenta.2005.04.007)

Zeghichi-Hamri S, de Lorgeril M, Salen P, Chibane M, de Leiris J, Boucher F \& Laporte F 2010 Protective effect of dietary n-3 polyunsaturated fatty acids on myocardial resistance to ischemia-reperfusion injury in rats. Nutrition Research 30 849-857. (doi:10.1016/j.nutres.2010.10.010)

Zhou SJ, Yelland L, McPhee AJ, Quinlivan J, Gibson RA \& Makrides M 2012 Fish-oil supplementation in pregnancy does not reduce the risk of gestational diabetes or preeclampsia. American Journal of Clinical Nutrition 95 1378-1384. (doi:10.3945/ajcn.111.033217)

Received 2 July 2013

First decision 29 July 2013

Revised manuscript received 5 September 2013

Accepted 10 September 2013 